\title{
Edad y crecimiento de Gobio lozanoi Doadrio \& Madeira, 2004 (Cypriniformes: Cyprinidae) en sectores fluviales de la cuenca del río Segura (SE península ibérica)
}

Fátima Amat-Trigo, Francisco J. Oliva-Paterna, David Verdiell-Cubedo, Ana Ruiz-Navarro \& Mar Torralva

Departamento de Zoología y Antropología Física. Facultad de Biología, Universidad de Murcia. 30100 Murcia, España.

\author{
Correspondencia \\ F. Amat-Trigo \\ E-mail: fatima.amat@um.es \\ TIf.:+34 868884961
}

Recibido: 25 octubre 2013

Aceptado: 28 noviembre 2013

Publicado on-line: 18 diciembre 2013

\section{Resumen}

El trabajo estudia la estructura de edad, longitud retrocalculada y tasa de crecimiento en poblaciones de Gobio lozanoi a lo largo de los principales ejes fluviales de la cuenca del río Segura (ríos Segura y Mundo). Se analizaron estos parámetros con individuos capturados en otoño de 2009 y en 19 localidades, así como su relación con variables ambientales (altitud, estado ecológico y conductividad). Las poblaciones reflejaron seis clases de edad $(0+a$ $5+)$ con escasas diferencias entre sexos y dominancia de edades intermedias $(2+$ y $3+)$. La estructura de edades mostró variaciones significativas en el gradiente con tendencia a presentar menor número de clases en localidades a menor altitud. La tasa de crecimiento se relacionó negativamente con la longevidad y mostró diferencias entre sectores fluviales. La tasa de crecimiento en la fase de transición a la madurez mostró tendencia a disminuir con el gradiente, pero también conforme decrece el estado ecológico y aumenta la conductividad.

Palabras clave: Tasa de crecimiento, Longitudes retrocalculadas, Estructura de edad, Gradiente longitudinal, Cyprinidae.

\begin{abstract}
Age and growth of Gobio lozanoi Doadrio \& Madeira, 2004 (Cypriniformes: Cyprinidae in fluvial sectors from the Segura river basin (SE Iberian Península)

The paper studies the age structure, back-calculated length and growth rate of Gobio lozanoi populations along the two main river axes of the Segura river basin (Segura and Mundo rivers). These parameters were analyzed from individuals captured in autumn 2009 and throughout 19 sampling locations, and their relationship with environmental variables (altitude, ecological status and conductivity). Populations showed six age classes $(0+$ to $5+)$ with few differences between sexes and dominance of intermediate ages $(2+$ and $3+)$. The age structure showed significant variations along the gradient with a tendency to narrower ranges of age in lower altitude locations. Growth rates showed a negative relationship with longevity and significant differences among fluvial sectors. The growth rate in the phase of transition to maturity showed a tendency to decrease with the gradient as well as the ecological state diminished and the water conductivity increased.
\end{abstract}

Key words: Growth rate, Back-calculated lengths, Age structure, Longitudinal gradient, Cyprinidae. 


\section{Introducción}

Gobio lozanoi (Doadrio \& Madeira 2004), es un ciprínido de pequeño tamaño de hábitos gregarios y bentónicos que se alimenta básicamente de macroinvertebrados (Oscoz et al. 2006). Es una especie común en tramos medios de los ríos donde los fondos son arenosos o de grava, aunque coloniza con facilidad otros tipos de hábitat, por lo que se puede encontrar en ambientes lénticos y someros como embalses (Doadrio et al. 2011). Se distribuye por toda la península ibérica y el sur de Francia. No obstante, solo se encuentra de forma nativa en las cuencas del Ebro y Bidasoa, teniendo carácter alóctono en el resto de cursos fluviales por los que se extiende ampliamente (Lobón-Cerviá et al. 1991, Doadrio \& Madeira 2004). La presencia de G. lozanoi en la cuenca hidrográfica del Segura es el resultado de la transferencia de agua a través del trasvase Tajo-Segura, aunque existen poblaciones de carácter puntual cuya presencia se relaciona con traslocaciones directas (Torralva et al. 2005). Este carácter de especie introducida en la cuenca del río Segura aumenta la importancia de obtener datos sobre su biología y estrategia de vida (Elvira 1998, Ribeiro et al. 2008). Son varios los estudios realizados sobre la especie en la península ibérica (Lobón-Cerviá \& Torres 1984, Lobón-Cerviá et al. 1991, Miñano et al. 2003), si bien, se centran en análisis temporales sobre determinadas poblaciones y siguen siendo escasos los conocimientos básicos sobre la estrategia de vida de la especie.

La heterogeneidad ambiental de los ríos mediterráneos ibéricos ofrece un marco muy útil para el estudio de la variabilidad entre poblaciones de peces continentales (Vila-Gispert \& Moreno-Amich 2001, Oliva-Paterna et al. 2003a, 2003b, Encina et al. 2006). La presencia de poblaciones de $G$. lozanoi en amplias secciones fluviales de la zona alta y media de la cuenca del río Segura (Torralva et al. 2005, Martínez-Morales et al. 2010) nos ofrece la posibilidad de estudiar la variabilidad espacial en parámetros de su crecimiento frente a los cambios ambientales a lo largo de gradientes fluviales de carácter longitudinal. Además, son relativamente escasos los estudios que describen los cambios en la biología poblacional de una especie fluvial a lo largo del gradiente longitudinal en dichos sistemas mediterráneos (Descroix et al. 2009, Tedesco et al. 2009, Castejón-Bueno et al.
2011). En el presente estudio, bajo la suposición de una variación en las poblaciones condicionada por el gradiente longitudinal, se pretende analizar la estrategia de crecimiento de G. lozanoi mediante el estudio de la estructura de edades, longitudes retrocalculadas y tasas de crecimiento de sus poblaciones, y evaluar las diferencias entre distintos sectores fluviales de la cuenca del río Segura con características ambientales diferentes.

\section{Material y Métodos}

El área de estudio se encuentra situada al sureste de la península ibérica y corresponde a la parte alta y media de la cuenca hidrográfica del Segura abarcando los dos ejes principales de la cuenca, el río Mundo y el propio río Segura. Esta zona se caracteriza por una elevada aridez, régimen hidrológico marcado por fuertes fluctuaciones naturales y un elevado nivel de regulación hídrica, todo ello con importantes efectos sobre las poblaciones y comunidades de peces (Torralva et al. 1997, 2005, Oliva-Paterna et al. 2003a, 2003b, Martínez-Morales et al. 2010, Verdiell-Cubedo et al. 2011). Se muestrearon un total de 19 localidades (Fig. 1), estableciendo sectores fluviales de estudio separados principalmente por grandes barreras físicas (presas) a lo largo de dos ejes longitudinales principales, río Segura $(\sim 160 \mathrm{~km})$ y río Mundo $(\sim 75$ $\mathrm{km})$. En el presente estudio, asumimos la existencia de un grado de conexión relativamente elevado entre individuos capturados en localidades de un mismo sector, mientras que asumimos una baja conexión con los capturados en sectores contiguos localizados aguas abajo. También existe una mayor homogeneidad a nivel de las características de macrohábitat entre las localidades de un mismo sector (Tabla 1).

Fueron capturados un total de 891 ejemplares de $G$. lozanoi entre finales de octubre y noviembre de 2009, de modo que se evitaron posibles efectos relacionados con el desarrollo gonadal y la reproducción de la especie sobre los patrones de crecimiento (Miñano et al. 2003). La técnica utilizada para la obtención de las muestras fue la pesca eléctrica (Modelo ERREKA III, 4200 W, 200-350 $\mathrm{V}, 2-3 \mathrm{~A}$ ), siguiendo el procedimiento descrito en la normativa CEN Water Analysis-Fishing with electricity (CEN 2003). Tras la captura, los individuos fueron anestesiados, conservados en formaldehido $10 \%$ y transportados al laboratorio donde 


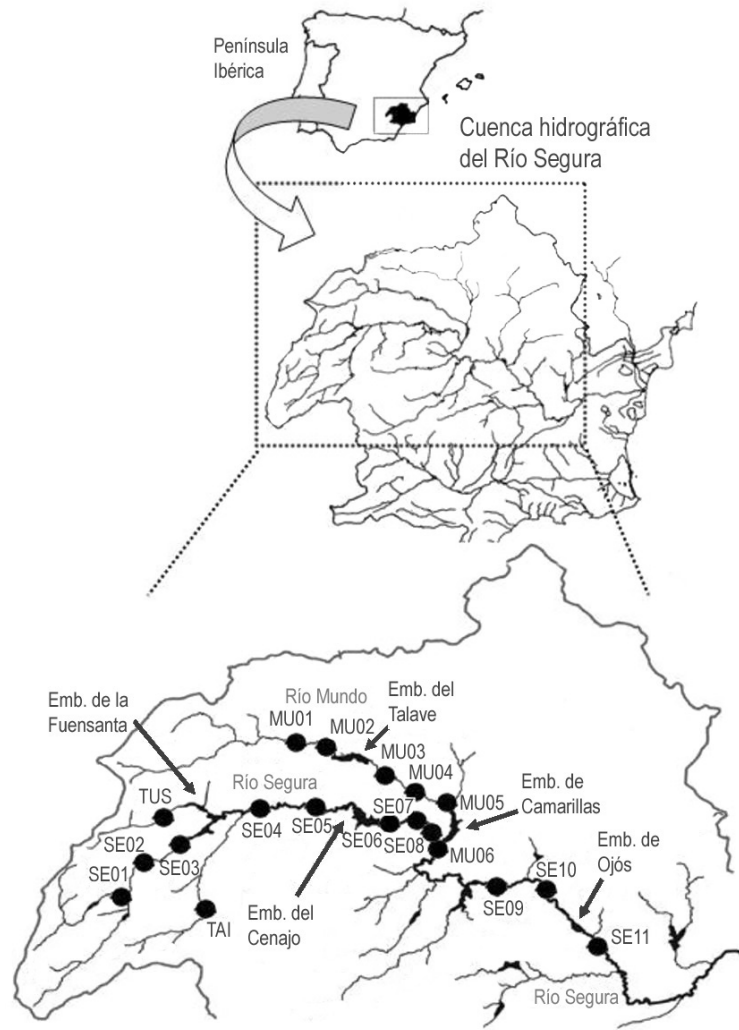

Figura 1. Cuenca del río Segura y localización de las localidades muestreadas. TAI $=$ Río Taibilla. TUS $=$ Río Tus. $\mathrm{MU}=$ Río Mundo. SE = Río Segura. Se marcan los principales embalses. Figure 1. Segura River Basin and location of the sampling sites. $\mathrm{TAI}=$ Taibilla River. $\mathrm{TUS}=$ Tus River. $\mathrm{MU}=$ Mundo River. $\mathrm{SE}=$ Segura River. Main reservoirs are marked. fueron procesados. De la totalidad de individuos se obtuvo la longitud furcal $(L f \pm 1 \mathrm{~mm})$ y se determinó el sexo mediante observación directa de las gónadas. Además, de cada ejemplar se obtuvo una muestra de escamas (extraídas del flanco izquierdo entre el inicio de la aleta dorsal y la línea lateral) para la determinación de la edad y el análisis de retrocálculo. Durante el proceso de lectura de escamas fueron desestimados ejemplares no válidos para el análisis (reabsorción de escamas o no distinción de annuli), reduciéndose el número total de individuos para el estudio de retrocálculo a un total de 575.

La estructura de las poblaciones se estableció mediante el análisis de las distribuciones de frecuencia por cada clase de edad para el total de individuos, machos y hembras en cada sector de estudio. Los individuos indeterminados fueron incluidos tanto dentro del conjunto de los machos como en el de las hembras para la construcción de los gráficos y los análisis estadísticos. Para establecer las longitudes de las edades pretéritas se siguió el criterio de Bagenal \& Tesch (1978):

$$
\mathrm{Ln}=[(\mathrm{Rn} / \mathrm{Rt})(\mathrm{Lf}-\mathrm{a})]+\mathrm{a}
$$

donde $L f$ es la longitud furcal del pez en el momento de captura, $L n$ es la longitud furcal a la edad $n, R t$ es

\begin{tabular}{|c|c|c|c|c|c|c|}
\hline Código & Denominación & $\mathrm{n}$ & Altitud (m) & $\begin{array}{c}\text { Estado } \\
\text { Ecológico }\end{array}$ & $\begin{array}{c}\text { Conductividad } \\
(\mu \mathrm{S} / \mathrm{cm})\end{array}$ & Sectores \\
\hline TUS & Río Tus & 21 & 809 & 1 & 432,33 & Tributarios \\
\hline TAl & Las Claras & 21 & 640 & 2 & 566,33 & Tributarios \\
\hline MU01 & Los Vizcainos & 37 & 580 & 2 & 597,33 & Sector 1 \\
\hline MU02 & Azud Liétor & 49 & 540 & 2 & 619,33 & Sector 1 \\
\hline MU03 & Puente Blas García & 35 & 430 & 3 & 674,33 & Sector 2 \\
\hline MU04 & Casas de los Pinos & 29 & 396 & 3 & 724,33 & Sector 2 \\
\hline MU05 & Cola Embalse Camarillas & 32 & 354 & 3 & 1303,33 & Sector 2 \\
\hline MU06 & Bajo Presa Camarillas & 17 & 350 & 4 & 1108,67 & Sector 6 \\
\hline SE01 & Las Juntas-Salto de Miller & 57 & 700 & 1 & 364,00 & Sector 3 \\
\hline SE02 & Camping & 35 & 685 & 1 & 386,67 & Sector 3 \\
\hline SE03 & Cola Embalse Fuensanta & 25 & 650 & 1 & 390,33 & Sector 3 \\
\hline SE04 & Bajo Presa Fuensanta-Peñarrubia & 32 & 460 & 1 & 356,00 & Sector 4 \\
\hline SE05 & Puente Hijar & 14 & 432 & 1 & 401,33 & Sector 4 \\
\hline SE06 & Bajo Cenajo & 12 & 363 & 2 & 446,67 & Sector 5 \\
\hline SE07 & Azud Salmerón- Azud del Rey & 33 & 325 & 2 & 491,67 & Sector 5 \\
\hline SE08 & Las Minas & 23 & 306 & 2 & 579,33 & Sector 6 \\
\hline SE09 & Río Muerto & 21 & 200 & 2 & 860,67 & Sector 7 \\
\hline SE10 & Azud Abarán & 15 & 148 & 2 & 1139,33 & Sector 7 \\
\hline SE11 & Azud Ulea & 27 & 112 & 4 & 1157,33 & Sector 8 \\
\hline
\end{tabular}

Tabla 1. Listado de las localidades muestreadas en el presente estudio. Se muestran los individuos capturados de Gobio lozanoi (n), los valores de las variables ambientales empleadas en su caracterización y sector al que pertenecen. La categorización de las variables se presenta en el texto.

Table 1. List of localities sampled in this study. It show individuals of Gobio lozanoi captured (n), the environmental variables values used in their characterization and their corresponding sector. The categorization of the variables is presented in the text. 
el radio de la escama a la edad $n$ y $a$ la ordenada en el origen de la relación lineal obtenida entre el radio total de la escama $(R t)$ y la longitud furcal $(L f)$. Se realizaron análisis de covarianza (ANCOVAs, $p<0,05)$ con los datos transformados logarítmicamente $(\log 10)$, para determinar la presencia de diferencias significativas en dicha relación lineal entre machos y hembras, con el objetivo de elaborar las tablas del retrocálculo conjuntas o por sexos separados. También se analizó la presencia de diferencias entre los sectores de estudio (en estos análisis no fueron incluidos los individuos indeterminados). Finalmente, se utilizó la prueba $t$ de Student $(p<0,05)$ para determinar las posibles diferencias entre las longitudes retrocalculadas a la edad $1+(\mathrm{LI}), 2+$ (LII) y $3+$ (LIII) entre machos y hembras.

El cálculo de las tasas instantáneas de crecimiento en longitud $(g)$ se realizó según Wootton (1998):

$$
\mathrm{g}=\left(\ln \mathrm{Lt}_{2}-\ln \mathrm{Lt}_{1}\right) /\left(\mathrm{t}_{2}-\mathrm{t}_{1}\right)
$$

donde $L t_{2}$ es la longitud media retrocalculada final, $L t_{l}$ es la longitud media retrocalculada inicial y $t_{2}-t_{l}$ es el periodo de tiempo transcurrido (ciclo anual completo en nuestro estudio). Se realizaron pruebas de la varianza de un factor (ANOVAs, $p<$ $0,05)$ para determinar la existencia de diferencias significativas entre lo que hemos denominado tasa de crecimiento de inmaduros (tasa de crecimiento entre la transición de LI a LII; $g_{1}$ ) y tasa de crecimiento de transición a la madurez (tasa de crecimiento entre la transición de LII a LIII; $g_{2}$ ). Las edades de primera madurez establecidas para la especie en la cuenca del río Segura se presentan en Miñano et al. (2003). Las diferencias entre sectores y localidades de las longitudes retrocalculadas LI, LII y LIII para el total de los individuos, machos y hembras se analizaron mediante ANOVAs o mediante la prueba de Kruskal-Wallis ( $p<$ $0,05)$ cuando no se obtuvo homogeneidad en las varianzas (prueba de Levene).

Para el estudio de la relación entre el crecimiento y las características de hábitat en los gradientes longitudinales, únicamente se consideraron descriptores a una escala de hábitat amplia: altitud sobre el nivel del mar, estado ecológico según DMA y obtenido del Estudio general sobre la Demarcación Hidrográfica del Segura (CHS 2007) [categorías: Muy bueno (1), Bueno (2), Moderado (3) y Deficiente (4)], y la conductividad del agua $( \pm 0,01 \mu \mathrm{S} / \mathrm{cm})$. El estado ecológico es determinado en función de indicadores biológicos, hidromorfológicos y fisicoquímicos, siendo los dos últimos valorados en relación a su capacidad de afectar a los indicadores biológicos. Se obtuvieron índices de correlación de Pearson para variables cuantitativas e índices de Spearman para variables categóricas ordinales (estado ecológico) $(p<0,05)$.

\section{Resultados}

\section{Estructura de edades, tallas y tasas de crecimiento}

La estructura por edades de las poblaciones de estudio en cada sector fluvial se presenta en la Figura 2. Se observa un máximo de seis clases de edad $(0+$ a $5+)$, tanto para hembras como machos. No obstante, únicamente el Sector 1 ha mostrado la presencia de 6 clases de edad (Fig. 2), mientras que los sectores que menos clases presentan son los Sectores 7 y 8 (únicamente tres clases de edad). El mayor número de ejemplares capturados en ambos sexos fueron los pertenecientes a la clase $2+$, conformando el $42,3 \%$ de las capturas de los machos y $41,9 \%$ en las hembras. De forma general, se observa un dominio de individuos pertenecientes a las clases de edad $2+$ y $3+$ en la mayoría de los sectores (Fig. 2). En términos ge-

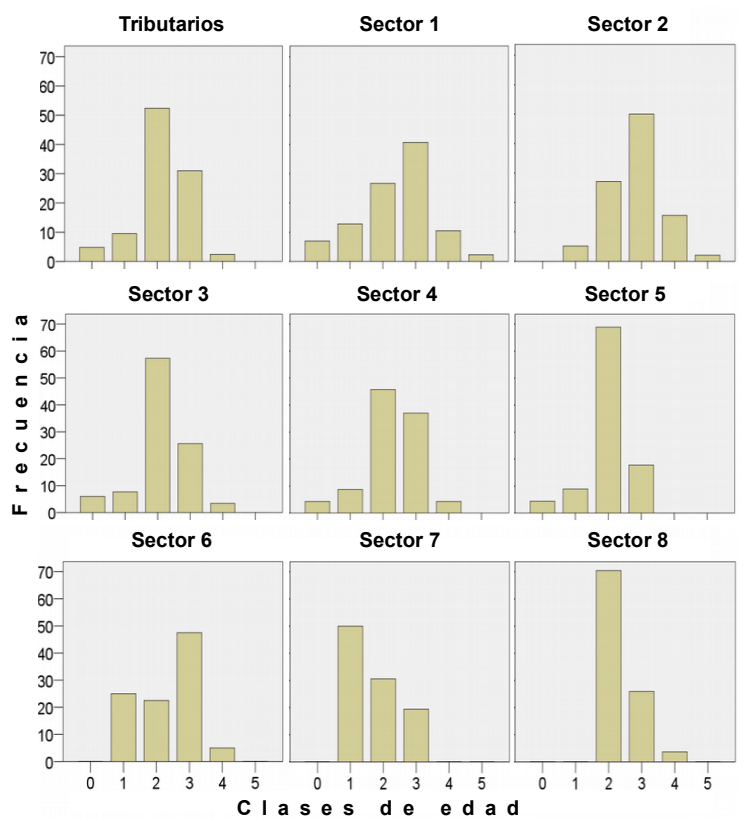

Figura 2. Estructura de edades de Gobio lozanoi para cada sector estudiado.

Figure 2. Age structure of Gobio lozanoi at each studied sector. 
nerales, las poblaciones con una estructura amplia y mayor número de clases de edad son escasas y se corresponden con los sectores localizados en las partes altas de la cuenca, mientras que aquellas localizadas a una menor altitud presentan un rango de clases de edad más estrecho.

La $L f$ mínima registrada ha sido de $26 \mathrm{~mm}$ para indeterminados, $37 \mathrm{~mm}$ para machos y 32 $\mathrm{mm}$ en hembras; las longitudes máximas registradas han sido de $113 \mathrm{~mm}(L f)$ para machos y 121 $\mathrm{mm}(L f)$ para hembras.

La relación entre la longitud furcal $(L f)$ y el radio total $(R t)$ de la escama resultó lineal para el total de los individuos $\left(L f=11,456+35,831 \mathrm{Rt} ; \mathrm{F}_{(1,}\right.$, $\left.{ }_{573)}=5488,13 ; p<0,001\right)$. Esta relación no mostró diferencias significativas entre sexos dentro de los sectores, así como entre los diferentes sectores (Tabla 2). Por ello, fueron tratados de forma conjunta los datos referentes a ambos sexos y se ha trabajado con una única ecuación de retrocálculo:

$$
\operatorname{Ln}=[(\mathrm{Rn} / \mathrm{Rt}) *(\mathrm{Lf}-10,885)]+10,885
$$

donde el valor 10,885 corresponde al punto de corte $a$.

En la mayoría de los sectores, no se detectaron diferencias significativas entre sexos para las longitudes retrocalculadas de las clases de edad 1+, $2+$ y $3+$ (LI, LII y LIII en Tabla 3), de este modo, se ha trabajado con una única tabla de retrocálculo por sector incorporando individuos de ambos sexos (Tabla 4). Las tablas de retrocálculo también nos confirman las estructuras de edades presentadas en el epígrafe anterior.

El rango de la longitud media retrocalculada para cada clase de edad se muestra en la Tabla 5. El sector que menor media ha mostrado en la longitud retrocalculada a la edad $1+$ ha sido el de Tributarios y el de mayor corresponde con el Sector 2. Las medias más elevadas de las longitudes retrocalculadas para las edades $2+$ y $3+$ se corres-

\begin{tabular}{cccccc}
\hline Sectores & \multicolumn{2}{c}{ Log Rt } & \multicolumn{2}{c}{ a intercept } & gl \\
\hline Tributarios & $\mathrm{F}=0,476$ & $p=0,496$ & $\mathrm{~F}=0,560$ & $p=0,461$ & $(1,30)$ \\
Sector 1 & $\mathrm{F}=0,836$ & $p=0,364$ & $\mathrm{~F}=0,052$ & $p=0,820$ & $(1,72)$ \\
Sector 2 & $\mathrm{F}=0,436$ & $p=0,511$ & $\mathrm{~F}=0,472$ & $p=0,494$ & $(1,85)$ \\
Sector 3 & $\mathrm{F}=0,686$ & $p=0,410$ & $\mathrm{~F}=1,680$ & $p=0,199$ & $(1,86)$ \\
Sector 4 & $\mathrm{F}=2,140$ & $p=0,151$ & $\mathrm{~F}=2,179$ & $p=0,148$ & $(1,44)$ \\
Sector 5 & $\mathrm{F}=0,705$ & $p=0,407$ & $\mathrm{~F}=1,140$ & $p=0,293$ & $(1,40)$ \\
Sector 6 & $\mathrm{F}=2,202$ & $p=0,147$ & $\mathrm{~F}=1,964$ & $p=0,170$ & $(1,37)$ \\
Sector 7 & $\mathrm{F}=1,562$ & $p=0,222$ & $\mathrm{~F}=0,841$ & $p=0,367$ & $(1,31)$ \\
Sector 8 & $\mathrm{F}=2,141$ & $p=0,161$ & $\mathrm{~F}=1,487$ & $p=0,238$ & $(1,22)$ \\
\hline Total & $\mathrm{F}=0,112$ & $p=0,738$ & $\mathrm{~F}=0,017$ & $p=0,897$ & $(1,447)$ \\
\hline
\end{tabular}

Tabla 2. Resultados de los ANCOVA realizados para determinar diferencias entre sexos en la relación Longitud furcal-Radio total de la escama dentro de cada sector y para el total de individuos. El Radio total (Rt) es la covariable, el sexo es el factor fijo y los estadísticos corresponden al contraste del punto de corte.

Table 2. Results of ANCOVA tests for the comparison of the Furcal length-Total scale radius relationships between sexes at each sector. Total radius (Rt) is the covariate, sex is the fixed factor and the statistics correspond to the a-intercept comparisons.

\begin{tabular}{cllllll}
\hline Sectores & \multicolumn{2}{c}{ LI } & \multicolumn{2}{c}{ LII } & \multicolumn{1}{c}{ LIII } \\
\hline Tributarios & $t=-1,423$ & $p=0,164$ & $t=-0,884$ & $p=0,384$ & $t=0,180$ & $p=0,860$ \\
Sector 1 & $t=-1,167$ & $p=0,247$ & $t=-3,001^{*}$ & $p=0,004$ & $t=-2,066^{*}$ & $p=0,045$ \\
Sector 2 & $t=-1,055$ & $p=0,294$ & $t=-1,435$ & $p=0,155$ & $t=0,769$ & $p=0,445$ \\
Sector 3 & $t=1,494$ & $p=0,139$ & $t=2,779^{*}$ & $p=0,007$ & $t=-2,036^{*}$ & $p=0,050$ \\
Sector 4 & $t=-1,885$ & $p=0,066$ & $t=-0,873$ & $p=0,388$ & $t=-0,909$ & $p=0,376$ \\
Sector 5 & $t=0,375$ & $p=0,710$ & $t=0,240$ & $p=0,812$ & $t=0,526$ & $p=0,621$ \\
Sector 6 & $t=1,921$ & $p=0,62$ & $t=1,850$ & $p=0,075$ & $t=0,796$ & $p=0,436$ \\
Sector 7 & $t=-1,572$ & $p=0,126$ & $t=-0,302$ & $p=0,767$ & $t=-0,195$ & $p=0,853$ \\
Sector 8 & $t=-0,025$ & $p=0,980$ & $t=0,689$ & $p=0,497$ & $t=-1,005$ & $p=0,354$ \\
\hline Total & $t=-1,004$ & $p=0,316$ & $t=-0,546$ & $p=0,585$ & $t=-1,263$ & $p=0,208$ \\
\hline
\end{tabular}

Tabla 3. Resultados de la prueba t de Student para determinar diferencias significativas en las longitudes retrocalculadas LI, LII y LIII entre sexos dentro de cada sector. Se ha asumido homogeneidad de varianzas. $(* p<0,05)$.

Table 3. Results of the t-Student test to determine significant differences in the back-calculated lengths LI, LII and LIII between sexes. Homogeneity of variances is assumed. $(* p<0,05)$. 


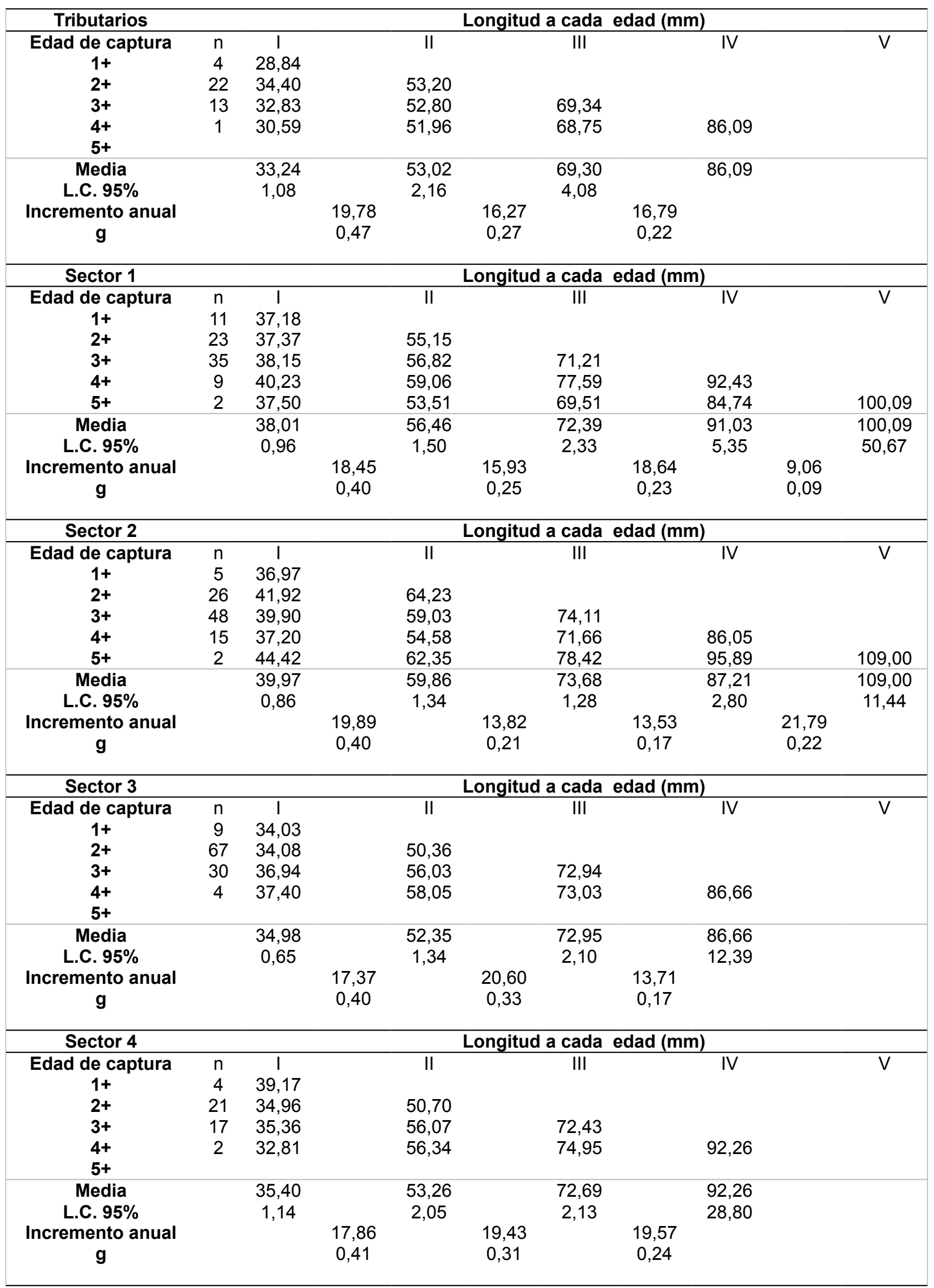

Tabla 4. Longitudes medias retrocalculadas ( $\mathrm{mm}$ ), incremento anual del crecimiento y tasas instantáneas de crecimiento ( $g$ ) de Gobio lozanoi en cada sector. Los números romanos indican las edades a las que han sido retrocalculadas las longitudes.

Table 4. Mean back-calculated lengths $(\mathrm{mm})$, anual growth increment and instantaneous growth rates $(\mathrm{g})$ of Gobio lozanoi at each sector. Roman numbers indicate ages of back-calculated lengths. 


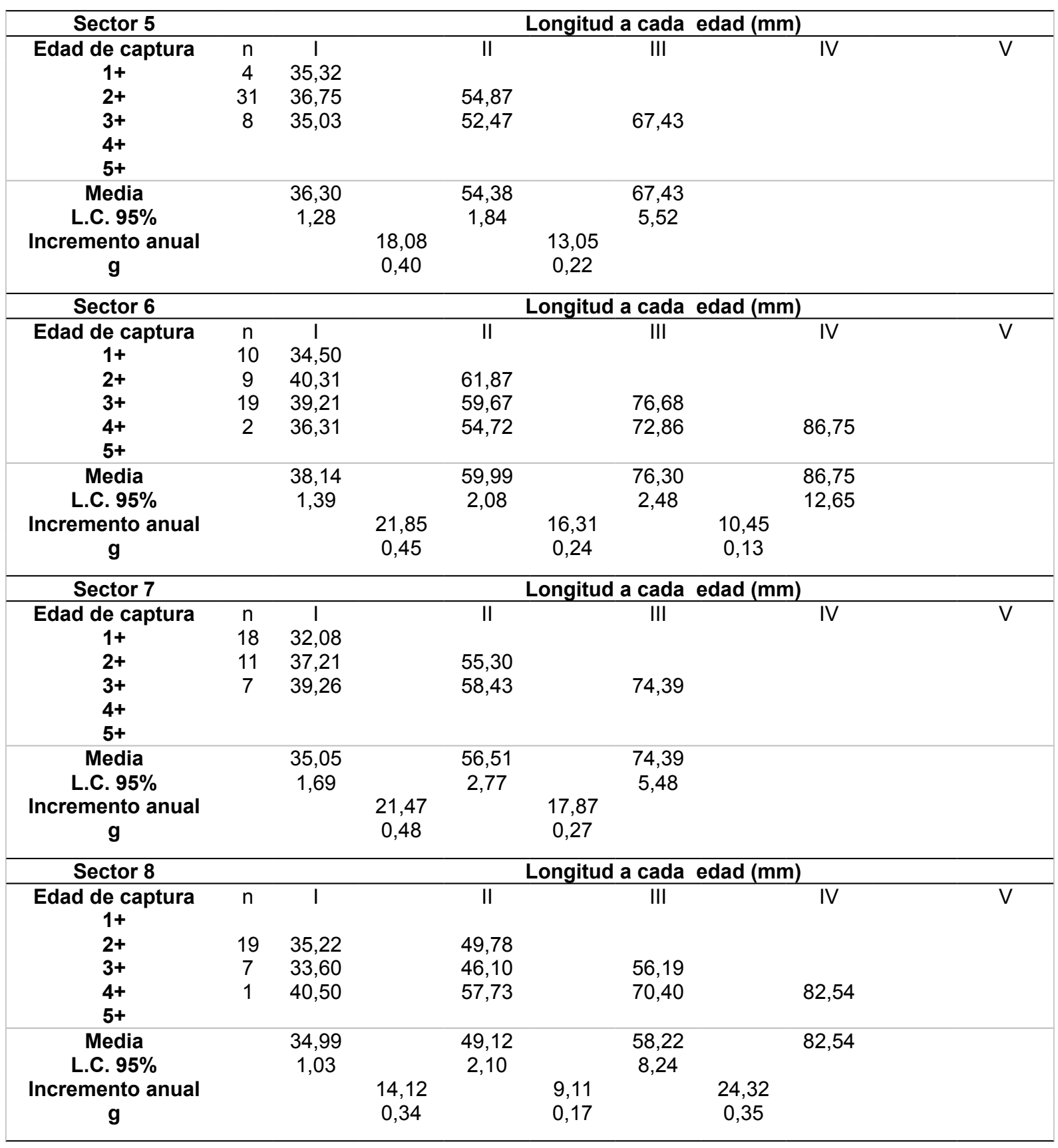

Tabla 4 (continúa). Longitudes medias retrocalculadas $(\mathrm{mm})$, incremento anual del crecimiento y tasas instantáneas de crecimiento (g) de Gobio lozanoi en cada sector. Los números romanos indican las edades a las que han sido retrocalculadas las longitudes.

Table 4 (continues). Mean back-calculated lengths ( $\mathrm{mm}$ ), anual growth increment and instantaneous growth rates (g) of Gobio lozanoi at each sector. Roman numbers indicate ages of back-calculated lengths.

\begin{tabular}{|cc|}
\hline Clase de Edad & Rango de Longitud media retrocalculada $(\mathbf{m m})$ \\
\hline $1+$ & $31,1-40,9$ \\
$2+$ & $47,0-62,1$ \\
$3+$ & $50,0-78,8$ \\
$4+$ & $82,5-121,1$ \\
\hline
\end{tabular}

Tabla 5. Rangos de la longitud media retrocalculada para cada clase de edad en la totalidad de sectores.

Tabla 5. Ranges of mean back-calculated lengths for each age class overall of sectors. 
ponden al Sector 6 y las más bajas aparecen en el Sector 8 .

De forma general, las tasas instantáneas de crecimiento $(g)$ han mostrado una clara relación con la longevidad de los individuos, presentando los ejemplares más jóvenes tasas más elevadas y descendiendo éstas a medida que avanzan en edad. La tasa de crecimiento dentro de individuos inmaduros $\left(g_{1}\right)$ oscila entre $34 \%$ y $48 \%$, mientras que la tasa de crecimiento en transición a la madurez $\left(g_{2}\right)$ oscila entre el $17 \%$ y $33 \%$ (Tabla 4$)$. El Sector 7 muestra el valor más elevado para la tasa de crecimiento de inmaduros y el Sector 3 para la tasa de crecimiento de transición a la madurez, mientras que el Sector 8 es el que presenta los valores más bajos para ambas tasas. La variación tanto en las longitudes retrocalculadas, así como en las tasas de crecimiento de peces con una edad superior a $3+$, ha resultado bastante elevada. Este patrón puede estar relacionado con la notable disminución del tamaño muestral de individuos con edades avanzadas. De este modo, no se han tenido en cuenta en el análisis para estudiar las posibles relaciones con la variación ambiental en el eje longitudinal.

\section{Variación longitudinal y relaciones con las variables ambientales}

Las tasas de crecimiento promedio $\left(g_{1}\right.$ y $\left.g_{2}\right)$ y las longitudes medias retrocalculadas (LI, LII y LIII), estimadas en función de los sectores establecidos a lo largo de los dos ejes de estudio, muestran diferencias significativas (ANOVA, $g_{1}: \mathrm{F}_{(8,442)}=$ $\left.5,134, p<0,001 ; \quad g_{2}: \mathrm{F}_{(8,213)}=2,185, p=0,03\right)$ (Kruskal-Wallis, LI $\mathrm{H}=112,65, \mathrm{gl}=8, p<0,001$; LII $\mathrm{H}=99,43, \mathrm{gl}=8, p<0,001$; LIII $\mathrm{H}=30,05, \mathrm{gl}=$ $8, p<0,001)$.

Las longitudes medias retrocalculadas para las clases de edad $1+, 2+$ y $3+$ frente a las variables ambientales no muestran ningún patrón de variación claro (Fig. 3). Únicamente se ha encontrado una correlación significativa positiva entre la longitud retrocalculada a la edad $1+\mathrm{y}$ el estado ecológico (Spearman: $\mathrm{r}=0,54 ; p=0,017$ ), indicando una mayor longitud retrocalculada observada en los individuos $1+$ conforme peor es el estado ecológico.

La tasa de crecimiento de inmaduros es superior a la de transición a la madurez en todas las localidades (Fig. 4). En relación a las variables ambientales consideradas, las tasas de crecimiento no muestran patrones de variación significativos en el gradiente longitudinal (Fig. 4). Frente a la altitud, muestran una cierta tendencia a la disminución conforme descendemos desde las localidades de cabecera (Fig. 4). La tasa de crecimiento de transición a la madurez $\left(g_{2}\right)$ presentó una correlación positiva con la altitud en ambos ejes de estudio (Pearson: Eje Segura, $\mathrm{r}=0,82 ; p=0,001$; Eje Mundo, $\mathrm{r}=0,76 ; p=0,007)$, mientras que para los inmaduros $\left(g_{1}\right)$ únicamente mostró correlación significativa en el eje del río Mundo (Pearson: $\mathrm{r}=$ $0,60 ; p=0,038)$. Frente al estado ecológico y la conductividad, el comportamiento de las tasas de crecimiento es similar (cierta tendencia a disminuir conforme decrece el estado ecológico y aumenta la conductividad) (Fig. 4). La tasa de crecimiento de transición a la madurez presentó una correlación negativa con el estado ecológico en el río Segura (Spearman: $\mathrm{r}=-0,70 ; p=0,012$ ) y en inmaduros una correlación negativa con la conductividad en el río Mundo (Pearson: $\mathrm{r}=-0,71 ; p=$ 0,010). En su conjunto, podemos decir que estos resultados indican que a peor estado ecológico y mayor conductividad menor es la tasa de crecimiento.

\section{Discusión}

Los resultados obtenidos muestran cierta variabilidad en la estructura por edades de las poblaciones de G. lozanoi analizadas a lo largo de gradientes longitudinales en la cuenca del río Segura. Se ha constatado la presencia de poblaciones con seis clases de edad, correspondientes en su mayoría a localidades de cabecera y partes altas, y poblaciones con únicamente tres clases de edad en sectores fluviales situados a menor altitud. La estructura por edades observada en las poblaciones de cabecera ha resultado similar a la obtenida en otros estudios sobre la especie en la península ibérica (Lobón-Cerviá et al. 1991, Miñano et al. 2003), donde los ejemplares más longevos correspondieron a la clase de edad 5+. No obstante, el número de clases de edad es superior al encontrado en otros ríos penínsulares, donde los ejemplares más longevos pertenecían a la clase 3+ (ríos Matarraña y Moros) o 4+ (río Jarama y embalse de Pinilla) (Lobón-Cerviá \& Torres 1983, Lobón-Cerviá et al. 1991). En sistemas fluviales del resto de Europa, las poblaciones de diversas especies del género Gobio Cuvier, 1816, próximas taxonómicamen- 

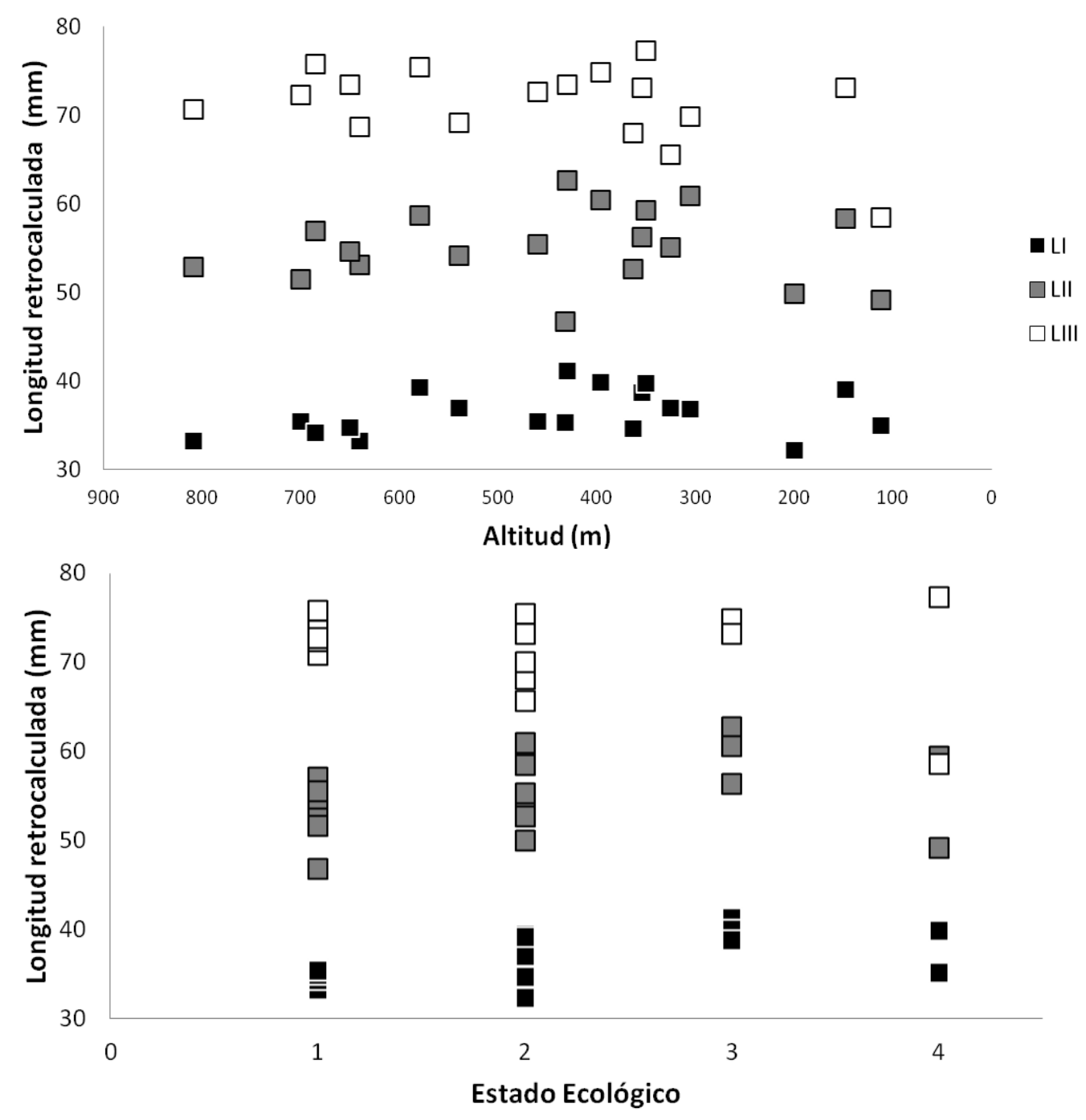

- $\mathrm{LI}$

$\square$ LII

$\square$ LIII

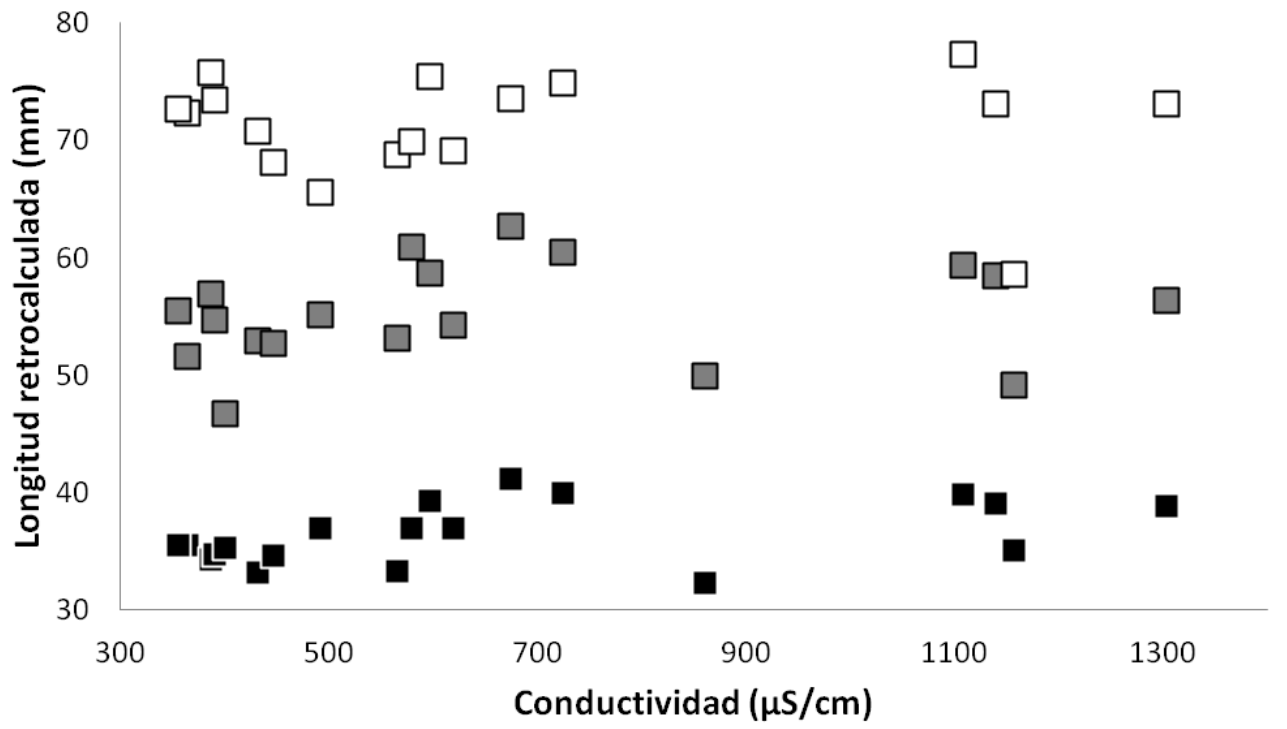

Figura 3. Relación entre las longitudes medias retrocalculadas $(\mathrm{mm})$ para las clases de edad $1+, 2+$ y $3+$ (LI, LII, LIII) y las variables ambientales: altitud sobre el nivel del mar, estado ecológico y conductividad.

Figure 3. Relationship between back-calculated lengths mean $(\mathrm{mm})$ for age classes $1+, 2+$ and $3+$ (LI, LII, LIII) and environmental variables: altitude above sea level, ecological status and conductivity. 

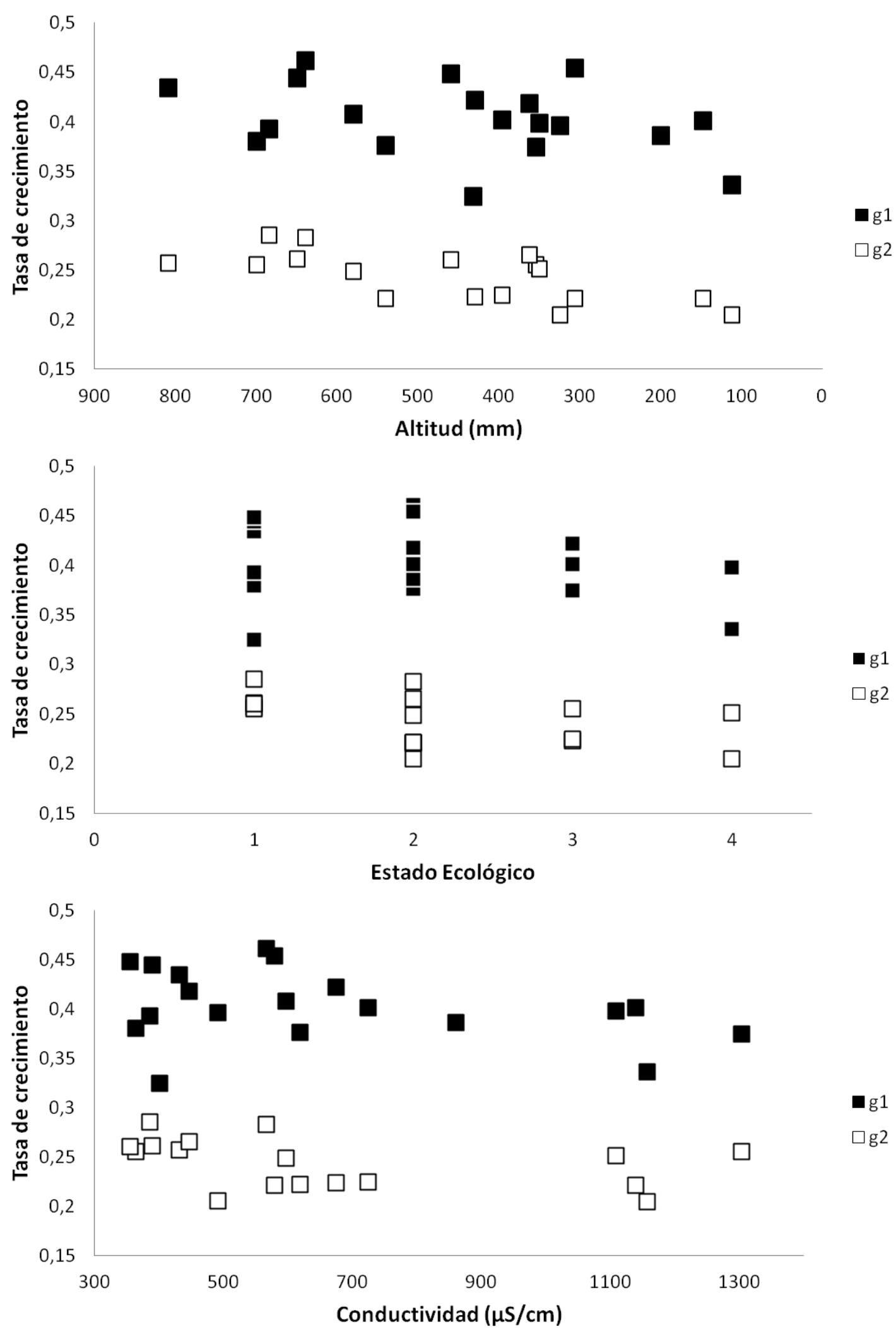

Figura 4. Relación entre las tasas de crecimiento de inmaduros $\left(g_{1}\right)$ y de transición a la madurez $\left(g_{2}\right)$ y las variables ambientales: altitud sobre el nivel del mar, estado ecológico y conductividad.

Figure 4. Relationship between growth rates of immature $\left(g_{1}\right)$ and of the transition to mature fish $\left(g_{2}\right)$ and environmental variables: altitude above sea level, ecological status and conductivity. 
te a la especie peninsular, llegan a mostrar estructuras con hasta ocho clases de edad (Kennedy \& Fitzmaurice 1972). En nuestro estudio, las clases de edad predominantes fueron $2+$ y $3+$, siendo dominantes los individuos $3+$ en los sectores del río Mundo, mientras que en las localidades del Segura y los Tributarios han mostrado un dominio de individuos con edades inferiores o iguales a $2+$. Este patrón también se presenta en otras poblaciones analizadas en el río Segura (Miñano et al. 2003) y en otros ríos penínsulares (Lobón-Cerviá et al. 1991). La presencia de pocas clases de edad es una característica propia de algunas especies de pequeños ciprínidos ibéricos (Granado-Lorencio 1992, Encina et al. 2006).

Los ejemplares de mayor longitud fueron capturados en las localidades de la zona alta y llegaron a alcanzar un máximo de $121 \mathrm{~mm}$ (longitud furcal), reflejando una longitud máxima similar a otros ríos peninsulares (Lobón-Cerviá \& Torres 1983, Lobón-Cerviá et al. 1991). Sin embargo, en el estudio realizado por Miñano et al. (2003) en la cuenca del Segura, no se obtuvieron peces con longitudes superiores a $101 \mathrm{~mm}$ durante un periodo aproximado de dos ciclos anuales completos. Las longitudes máximas descritas en la península ibérica no superan a las observadas en las poblaciones de Gobio gobio L., 1758 de algunos ríos europeos, que pueden albergar individuos de 160 y $170 \mathrm{~mm}$, y que habitan sistemas con dinámicas normalmente más estables que las presentes en nuestros sistemas ibéricos (Hartley 1947, Kennedy \& Fitzmaurice 1972, Mann 1980).

A pesar de la elevada variabilidad en la estructura de edades entre localidades, no se observa un gradiente claro de variación relacionado con el eje longitudinal. Esta variación puede ser debida, en parte, a la plasticidad en el crecimiento que la especie puede presentar en diferentes ambientes fluviales (Weatherley \& Gill 1987). No obstante, se ha detectado una dominancia de poblaciones con presencia de un mayor número de grupos de edad en las localidades de zonas altas, aspecto que podría indicar una mayor viabilidad de las mismas. Este hecho resulta comprensible teniendo en cuenta el carácter montano de la especie, que muestra preferencia por tramos de corriente moderada y aguas de mayor calidad (Doadrio 2002, Doadrio et al. 2011), que en la cuenca del Segura se localizan principalmente en zonas de mayor altitud. A su vez, la especie presenta un carácter no nativo en la Cuenca, siendo la zona alta de la misma la receptora de la invasión (Martínez-Morales et al. 2010). Este aspecto también podría tener cierto grado de relación con el estado actual de sus poblaciones (Ribeiro et al. 2008).

Las poblaciones presentaron una relación lineal entre la longitud furcal y el radio total de la escama como se ha obtenido en otros estudios realizados con la misma especie (Miñano et al. 2003) y con otras especies de ciprínidos peninsulares (Lobón-Cerviá \& Torres 1983, Miñano et al. 2000, Torralva et al. 1997). Sin embargo, no se detectaron diferencias significativas en las longitudes retrocalculadas entre sexos, aspecto que no coincide con lo observado en otros estudios sobre la especie (Lobón-Cerviá et al. 1991, Miñano et al. 2003).

La relación detectada entre las tasas de crecimiento y la longevidad de los individuos resultó similar a la descrita por Miñano et al. (2003) en un sector de la parte alta del Segura. Los ejemplares más jóvenes presentaron un elevado incremento de tamaño ( $42 \%$ de tasa de crecimiento promedio entre inmaduros de la clase $1+$ y $2+$ ), que desciende conforme los individuos son más longevos ( $25 \%$ de tasa de crecimiento promedio entre individuos $2+$ y $3+$, transición a la madurez). El decremento en la tasa de crecimiento conforme aumenta la longevidad concuerda con la estrategia de vida intermedia entre oportunista y periódica descrita para la especie por Vila-Gispert \& Moreno-Amich (2002). Además, tal aspecto, se acompaña del alcance de la madurez sexual a edades tempranas (Lobón-Cerviá et al. 1991). Este hecho puede verse aumentado en aquellos hábitats con estrés ambiental muy acusado donde la especie, además, realiza una fuerte inversión de energía en la reproducción con el fin de asegurar su supervivencia (Lobón-Cerviá et al. 1991, Miñano et al. 2003).

En nuestro estudio, no podemos concluir sobre la presencia de una relación lineal entre el gradiente longitudinal y los descriptores de la edad y crecimiento. No obstante, la tasa de crecimiento de transición a la madurez (tasa entre individuos $2+$ y $3+$ ), muestra una cierta tendencia a disminuir con dicho gradiente. En los cursos de ríos naturales es esperable que conforme avanzamos en el gradiente decrezca la frecuencia de rápidos y disminuya la velocidad del agua (Bouchard et al. 1998), además del incremento en la temperatura 
del agua conforme se reduce la altitud. En su conjunto, es esperable un decremento en la demanda de consumo energético de los peces asociado a la dinámica del caudal y, en consecuencia, un aumento de las tasas de crecimiento también favorecidas por el incremento de temperatura (Tedesco et al. 2009). No obstante, la disminución en las tasas de crecimiento en las zonas más bajas de la cuenca observada en nuestro estudio podría estar relacionada con una baja disponibilidad de alimento, la presencia de temperaturas del agua superiores al óptimo de crecimiento de la especie o factores relacionados con la competencia interespecífica (Weatherley \& Gill 1987). Nuestros resultados muestran que las localidades situadas a menor altitud presentan riberas predominantemente agrícolas, elevados valores de conductividad y peores estados ecológicos. Este decremento en la calidad del hábitat fluvial podría explicar el patrón observado en las tasas de crecimiento. En este sentido, efectos similares sobre otros parámetros relacionados con el crecimiento, como es la condición somática, ya se han observado en la propia especie (Amat-Trigo 2013) y otras especies de ciprínidos de la cuenca del río Segura (Oliva-Paterna 2003a, 2003b, Castejón-Bueno et al. 2011).

Por otro lado, nuestros resultados muestran ejemplares con una mayor talla retrocalculada a la edad 1+ en sectores con un estado ecológico más deficiente. Esto podría deberse a una respuesta adaptativa de la especie en un intento de alcanzar la madurez lo antes posible en un ambiente no óptimo para la misma. La talla óptima para maximizar la viabilidad poblacional depende de la fecundidad y supervivencia de cada clase de edad. En este contexto, la alta plasticidad interpoblacional en tácticas reproductoras y las características ambientales del área de estudio podrían repercutir indirectamente en el patrón de crecimiento de nuestra especie (Mann 1980). Además, las especies exóticas invasoras muestran una tendencia a expandir su rango de distribución una vez introducidas pudiendo establecerse en lugares no óptimos para las especies autóctonas (Ribeiro et al. 2008, Leunda 2010).

En conclusión, los parámetros biológicos analizados sobre el crecimiento de G. lozanoi en la cuenca del Segura resultaron muy heterogéneos dentro de cada sector o tramo fluvial de estudio. Aunque éstos estuvieron ligeramente relacionados con el gradiente longitudinal y altitudinal, otros factores ambientales no evaluados en el presente estudio (ej. disponibilidad trófica, competencia intra e interespecífica, etc.) podrían tener una influencia significativa sobre dichos parámetros de crecimiento. Por tanto, es recomendable profundizar en el estudio de la influencia de las condiciones de hábitat sobre la edad y crecimiento de la especie objetivo.

\section{Agradecimientos}

Agradecer a los miembros del grupo de investigación Zoología Básica y Aplicada a la Gestión y a la Conservación por su ayuda e interés en este trabajo. A la Fundación Séneca por la financiación del proyecto: "Efecto de la modificación antrópica del régimen hídrico sobre los peces epicontinentales nativos y exóticos de la cuenca del río Segura", que ha permitido la realización de este estudio. Parte de este se ha realizado en el contexto del Máster en Gestión de la Biodiversidad de Ambientes Mediterráneos de la Universidad de Murcia.

\section{Referencias}

Amat-Trigo F. 2013. Variación poblacional de Gobio lozanoi Doadrio \& Madeira, 2004 (Cypriniformes: Cyprinidae) en gradientes longitudinales de la cuenca del río Segura (SE Península Ibérica). Tesis de Licenciatura. Murcia (España): Universidad de Murcia.

Andreu-Soler A. 2008. La ictiofauna epicontinental de la Región de Murcia: distribución, problemática y propuestas para su conservación. Tesis Doctoral. Murcia (España): Universidad de Murcia.

Bagenal T \& Tesch FW. 1978. Age and Growth. En Methods for assessment of fish production in fresh waters (Bagenal T, ed.). London: Blackwell Scientific Publications.

Bouchard P, Chappaz R, Cavalli L \& Brun G. 1998. Influence of environmental variables on the growth of Leuciscus cephalus (Linnaeus 1766), in the River Durance, South-east France. Annales de limnologie 34: 193-200.

Castejón-Bueno D, Torralva M, Verdiell-Cubedo D, Martínez-Morales I, Ruiz-Navarro A \& Oliva-Paterna FJ. 2011. Variación en la condición de Luciobarbus sclateri (Günther, 1868) (Cypriniformes: Cyprinidae) a lo largo de gradientes longitudinales de la cuenca del río Segura. Anales de Biología 33: 41-52.

CEN document. 2003. Water quality-Sampling of fish with electricity. CEN/TC 230.

CHS. Confederación Hidrográfica del Segura-Ministerio de Medio Ambiente. 2007. Estudio general sobre la Demarcación Hidrográfica del Segura. Versión 4. Disponible en http://www.chsegura.es [Accedido el 
día 4 de Junio de 2013].

Descroix A, Desvilettes C, Martin P, Anneville O, Bec A \& Bourdier G. 2009. Feeding, growth and nutritional status of restocked salmon parr along longitudinal gradient of a large European river, the Allier. Ecology of Freshwater Fish 18 (2): 282-296.

Doadrio I. 2002. Atlas y Libro Rojo de los Peces Continentales de España. Madrid: CSIC Ministerio de Medio Ambiente.

Doadrio I \& Madeira MJ. 2004. A new species of the genus Gobio Cuvier, 1816 (Actynopterigii, Cyprinidae) from the Iberian Peninsula and southwestern France. Graellsia 60 (1): 107-116.

Doadrio I, Perea S, Garzón-Heydt P \& González JL. 2011. Ictiofauna Continental Española. Bases para su seguimiento. Madrid: Dirección General Medio Natural y Política Forestal. Ministerio de Medio Ambiente y Medio Rural y Marino.

Elvira B. 1995. Native and exotic freshwater fishes in Spanish river basins. Freshwater Biology 33: 103108.

Elvira B. 1998. Impact of introduced fish on the native freshwater fish fauna of Spain. En Stocking and Introduction of Fish (Cowx IG, ed.). United Kingdom: Hull International Fisheries Institute.

Encina L, Rodríguez A \& Granado-Lorencio C. 2006: The Iberian ichthyofauna: ecological contributions. Limnetica 25 (1-2): 349-368.

Granado-Lorencio C. 1992. Fish species ecology in Spanish Freshwater ecosystems. Limnetica 8: 255261.

Hartley PHT. 1947. The natural history of some British freshwater fishes. Proceedings of the Zoological Society of London 117: 129-206.

Kennedy M \& Fitzmaurice P. 1972. Some aspects of the biology of gudgeon Gobio gobio (L.) in Irish waters. Journal of Fish Biology 4: 425-440.

Leunda PM. 2010. Impacts of non-native fishes on Iberian freshwater ichthyofauna: current knowledge and gaps. Aquatic Invasions 5: 239-262.

Lobón-Cerviá J \& Torres S. 1984. On the growth and reproduction of two populations of gudgeon (Gobio gobio L.) in Central Spain. Acta Hydrobiology 1: 101115.

Lobón-Cerviá J, Montañés C \& De Sostoa A.1991. Influence of environment upon the life history of gudgeon, Gobio gobio (L.): a recent and successful colonizer of the Iberian Peninsula. Journal of Fish Biology 39: $285-300$.

Mann RHK. 1980. The growth and reproductive strategy of the gudgeon, Gobio gobio (L.), in two hard-water rivers in southern England. Journal of Fish Biology 17: 163-176.

Martínez-Morales I, Oliva-Paterna FJ, Verdiell-Cubedo D \& Torralva M. 2010. Inventario y estado de conservación de la fauna piscícola en la cuenca alta de río Segura (SE Península Ibérica). Anales de Biología 32: 47-58.
Miñano PA, Oliva-Paterna FJ, Fernández-Delgado C \& Torralva M. 2000. Edad y crecimiento de Barbus graellsii Steindachner, 1866 y Chondrostoma miegii, Steindachner, 1866 (Pisces, Cyprinidae) en el río Cinca (Cuenca Hidrográfica del Ebro, NE España). Miscel lània Zoològica 23 (2): 9-19

Miñano PA, Garcia-Mellado A, Oliva-Paterna FJ \& Torralva M. 2003. Edad, crecimiento y reproducción de Gobio gobio L. (Pisces, Cyprinidae) en un tramo regulado del río Segura (SE España). Animal Biodiversity and Conservation 26 (1): 67-76.

Oliva-Paterna FJ, Vila-Gispert A \& Torralva M. 2003a. Condition of Barbus sclateri from semiarid aquatic systems: habitat quality effects. Journal of Fish Biology 63: 699-709.

Oliva-Paterna FJ, Miñano PA \& Torralva M. 2003b. Habitat quality affects the condition of Barbus sclateri in Mediterranean semi-arid streams. Environmental $\mathrm{Bi}$ ology of Fishes 67: 13-22.

Oscoz J, Campos F \& Escala MC. 2003. Alimentación del gobio (Gobio gobio (L. 1758)) en el río Larraun (Navarra, N. España). Limnetica 22 (3-4): 77-84.

Ribeiro F, Elvira B, Collares-Pereira MJ \& Moyle PB. 2008. Life-history traits of non-native fishes in Iberian watersheds across several invasion stages: a first approach. Biological Invasions 10 (1): 89-102

Tedesco PA, Sagnes P \& Laroche J. 2009. Variability in the growth rate of chub Leuciscus cephalus along a longitudinal river gradient. Journal of Fish Biology 74: 312-319.

Torralva M, Puig MA \& Fernández-Delgado C. 1997. Effect of river regulation on the lifehistory patterns of Barbus sclateri in the Segura river basin (south-east Spain). Journal of Fish Biology 51: 300-311.

Torralva M, Oliva-Paterna FJ, Andreu A, Verdiell-Cubedo D, Miñano PA \& Egea A. 2005. Atlas de Distribución de los Peces Epicontinentales de la Región de Murcia. Murcia: Dirección General del Medio Natural. CARM.

Verdiell-Cubedo D, Oliva-Paterna FJ, Martínez-Morales I \& Torralva-Forero M. 2011. Efectos de la modificación antrópica de los regímenes hidrológicos naturales sobre poblaciones de ciprínidos bentónicos de la Península Ibérica. VII Congreso Ibérico de Gestión y Planificación del Agua. Febrero 2011, Talavera de la Reina.

Vila-Gispert A \& Moreno-Amich R. 2001. Fish condition analysis by a weighted least squares procedure: testing geographical differences of an endangered Iberian cyprinodontid. Journal of Fish Biology 58: 1658-1666.

Vila-Gispert A \& Moreno-Amich R. 2002. Life-history patterns of 25 species from European freshwater fish communities. Environmental Biology of Fishes 65: 387-400.

Weatherley AH \& Gill HS. 1987. The Biology of Fish Growth. Great Britain: Academic Press.

Wootton RJ. 1998. Ecology of Teleost Fishes (Springer, ed.). London. 